\title{
ASPECTOS NUTRICIONAIS E ANTROPOMÉTRICOS DE PORTADORES DE COLELITÍASE
}

Lutty Yoshimassa Shimabukuro, Helen Dayane Pereira Castro, Mariana Neves Soares, Sandra Cristina

Universidade do Oeste Paulista - UNOESTE, Curso de Nutrição, Presidente Prudente, SP. E-mail: lutty.shimabukuro@hotmail.com.

\section{RESUMO}

Este trabalho teve como objetivo analisar o perfil nutricional, antropométrico e qualidade alimentar de pacientes submetidos à colecistectomia. Foram avaliados através de medidas antropométricas e questionário de frequência alimentar cinquenta pacientes, de ambos os sexos, com idade acima de 18 anos, internados na clinica cirúrgica do Hospital Regional de Presidente Prudente e submetidos à colecistectomia. Os resultados foram analisados estatisticamente pelo teste binomial e teste qui-quadrado. Observou-se que $86 \%$ dos portadores de colelitiase eram do sexo feminino, e a idade com a maior prevalência foi entre 31 e 60 anos (66\%). Em relação ao IMC a maior prevalência foi em obesos (45\%). O questionário de frequência alimentar identificou um alto consumo de fatores de risco para a litogênese. Como conclusão, verifica-se uma maior prevalência de colelitiase no sexo feminino, idade entre 31 e 60 anos, obesos e com alto consumo de açúcar simples e gordura.

Palavras chave: Avaliação Nutricional, Hábito Alimentar, Litogênese, Nutrição, Vesícula Biliar.

\section{NUTRITIONAL AND ANTHROPOMETRIC ASPECTS OF CHOLELITHIASIS PATIENTS}

\begin{abstract}
This study aimed to analyze the nutritional, anthropometric and food quality profile of patients undergoing cholecystectomy. Fifty patients of both sexes, aged over 18 years, were admitted to the general surgical clinic of the Regional Hospital of Presidente Prudente and underwent cholecystectomy. Anthropometric measurements were performed and a food frequency questionnaire was applied. The results were statistically analyzed using the binomial and chisquare test. The results showed that $86 \%$ of the patients with cholelithiasis were female, and the age with the highest prevalence was between 31 and 60 years (66\%). Regarding BMI, the highest prevalence was in obese individuals (45\%). The food frequency questionnaire identified a high consumption of risk factors for lithogenesis. In conclusion, the results indicated a higher prevalence of female cholelithiasis, aged between 31 and 60 years, obese and with high consumption of simple sugar and fat.
\end{abstract}

Keywords: Nutritional Evaluation, Feeding Habits, Lithogenesis, Nutrition, Gallbladder. 


\section{INTRODUÇÃO}

A prevalência desta doença nos Estados Unidos da América (EUA) é de mais de 25 milhões de casos com pelo menos um milhão de novos casos por ano. Tal enfermidade é muito comum em países ocidentais, notando-se maior incidência em mulheres e idosos ${ }^{1}$.

No Brasil, embora os estudos ainda sejam escassos e relativamente limitados, Coelho et al. ${ }^{2}$ avaliou, em necropsia, 1303 vesículas e encontrou uma ocorrência de 7,8\%, no qual a maior incidência foi em mulheres (5,3\%) e menor em homens (3,9\%). Em outro estudo, Torres et. al. ${ }^{3}$ analisou o resultado de 500 ultrassonografias que buscavam o esclarecimento de sintomas não relacionados à vesícula ou a via biliar e encontrou cálculos biliares em $92(18,4 \%)$ pacientes, sendo que $16 \%$ eram homens e $20,8 \%$ eram mulheres.

Colelitíase caracteriza-se por ser uma agregação incomum de componentes da bile ${ }^{4,5}$. Existem três tipos de cálculos biliares: cálculos de colesterol, cálculos pigmentados e cálculos mistos. Os de colesterol e os mistos quando somados são responsáveis por $80 \%$ dos casos. A diferença entre eles é que o primeiro é formado em $70 \%$ por colesterol e o segundo contém quantidades significativas de pigmentos (por exemplo, a bilirrubina). Os pigmentados são subdivididos em negros e marrons e são formados por sais de cálcio, bilirrubinas e proteínas ${ }^{6}$.

Dentre os fatores de risco para o desenvolvimento da colelitíase, grande parte possui forte relação com fatores relacionadas à nutrição, como dietas restritivas; jejum prolongado; alimentação parenteral total por estase biliar; cirurgia bariátrica pela rápida perda de peso; obesidade pela maior secreção de HmG-CoA redutase hepática, que tem como resultado a formação de uma bile supersaturada em colesterol. Com relação ao diabetes tipo II, a relação está entre a hiperinsulinemia e a neuropatia diabética, responsáveis por induzir a supersaturação de colesterol na bile e causar uma hipomobilidade da vesícula biliar. A dislipidemia, hipertrigliceridemia e baixas concentrações de $\mathrm{HDL}-\mathrm{c}$, possuem relação com a supersaturação da bile com colesterol, aumentando o risco para formação de cálculos de colesterol, bem como de cálculos pigmentados ${ }^{7,8,9}$.

Além das patologias supracitadas, o hábito alimentar inadequado se mostra como forte indutor da litogênese. Dentro deste contexto, a literatura relata que a baixa ingestão de fibras e a qualidade dos carboidratos consumidos estão diretamente relacionados ao risco de desenvolvimento de cálculos biliares ${ }^{7,8,9}$.

Os fatores que protegem contra a litogênese incluem a prática de exercício físico que está associada à diminuição da resistência à insulina e aumento da motilidade intestinal, reduzindo assim a absorção intestinal de colesterol da dieta. Ademais, os fatores de proteção dietéticos incluem a ingestão de uma dieta rica em fibras, que acelera o trânsito intestinal e a ingestão de alguns componentes da alimentação como cálcio, magnésio, vitamina $C$, consumo moderado de álcool ${ }^{2,8,9}$.

Como os fatores de risco e de proteção apresentam forte relação com doenças relacionadas à alimentação e ao padrão alimentar, justifica-se a elaboração de um estudo que relacione aspectos nutricionais, obesidade, diabetes e dislipidemia com litogênese biliar, para que se possa orientar melhor a população evitando assim problemas futuros.

Esse estudo teve como objetivo principal analisar o perfil nutricional, antropométrico e qualidade alimentar de pacientes submetidos à colecistectomia.

\section{METODOLOGIA}

A pesquisa foi iniciada após a aprovação do Comitê de Ética em Pesquisa da Universidade do Oeste Paulista (UNOESTE), № CAAE 59053816.5.0000.5515. Os sujeitos que concordaram em participar da pesquisa após serem informados sobre os objetivos do trabalho e os procedimentos aos quais seriam expostos, assinaram um termo de consentimento livre e esclarecido (TCLE) redigido para este fim. 
Este estudo caracterizou-se por ser do tipo observacional, descritivo com delineamento transversal e análises estatísticas qualitativas e quantitativas, desenvolvido na Clínica Cirúrgica do Hospital Regional de Presidente Prudente, SP.

A população alvo foi composta por amostra não probabilística intencional de 50 pacientes de ambos os sexos, sem distinção de raça e com idade superior a 18 anos, que possuíam cálculo na via vesícula biliar e que estavam deambulando, internados na Clínica Cirúrgica do Hospital Regional de Presidente Prudente. Foram excluídos da amostra pacientes que não atendiam aos requisitos citados anteriormente. A Clínica Cirúrgica possui capacidade para 36 pacientes internados quando todos os leitos estão ocupados.

Para iniciar a pesquisa, os pesquisadores fizeram uma busca nos prontuários dos pacientes internados na clínica cirúrgica no período de novembro de 2016 a junho de 2017, em busca de pacientes com diagnóstico de colelitíase. Depois de selecionados, os pesquisadores preencheram uma ficha elaborada pelos mesmos, contendo informações clínicas do paciente e classificação do IMC.

A avaliação do índice de massa corporal (IMC) teve como intuito, verificar sobrepeso e obesidade. Para esta avaliação, o peso atual foi verificado com auxílio da balança digital de chão da marca Plenna, com capacidade de $180 \mathrm{Kg}$ e precisão de $100 \mathrm{~g}$. A altura foi medida utilizando uma fita métrica com comprimento de 150 centímetros $(\mathrm{cm})$ e sensibilidade de $0,1 \mathrm{~cm}$, acoplada à parede. O cálculo do IMC que é um indicador simples de estado nutricional, foi calculado pelo peso atual $(\mathrm{Kg})$ /estatura $\left(\mathrm{m}^{2}\right)$, classificando-se o estado nutricional de acordo com os critérios da WHO (World Health Organization) ${ }^{10}$ para adultos e Lipschitz ${ }^{11}$ para idosos ( $\geq 60$ anos).

Para análise da alimentação, foi utilizado o questionário de frequência de consumo alimentar (QFA), construído e adaptado por Ribeiro et al. ${ }^{12}$ a partir de um questionário elaborado por Sichieri e Everhart ${ }^{13}$. O QFA é um instrumento relativamente rápido para ser aplicado, de baixo custo, sendo composto por sete grupos alimentares (cereais e leguminosas; óleos e gorduras; sobremesas e petiscos; carnes e ovos; leites e derivados; hortaliças e frutas; e bebidas; incluindo refrigerante e sucos industrializados). Cada um destes grupos alimentares possui entre 8 e 10 itens (ao todo são 52), exceto o grupo do leite e derivados que apresenta apenas três. Em cada item deve-se preencher a porção consumida de cada alimento (indicado um determinado número) bem como a frequência consumida por dia ou por semana.

Para este estudo, foram considerados os seguintes alimentos promotores de colelitíase: Leite Integral, logurte, Queijo amarelo (prato e mussarela), Requeijão, Ovo Frito, Peixe Enlatado em óleo (Sardinha/Atum), Embutidos (salsicha, linguiça, salame, presunto, mortadela), Carne Conservada no Sal (Bacalhau, carne seca/sol, pertences da feijoada), Visceras (Fígado, Rim, Coração), Molho para Salada, Bacon, Toucinho, Manteiga, Margarina, Maionese, Batat-Frita, Sanduíches, Pizza, Esfiha, Salgadinhos, Amendoim, Arroz Polido, Biscoito Salgado, Biscoito Doce, Bolos, Macarrão, Tubérculos (Cará, Mandioca, Batata, Inhame), Sorvete, Tortas, Geleias, Doces/Bala, Chocolates/Achocolatados/Bombom, Café com Açúcar, Suco Natural com Açúcar, Suco Artificial com Açúcar, Suco Artificial sem Açúcar e Refrigerante Normal, na seguinte frequência: de duas vezes ou mais por dia.

A principal limitação em relação à pesquisa esta relacionada à fidedignidade dos dados coletados, pois o trabalho com indivíduos depende da veracidade de suas respostas.

Os dados foram submetidos à análise estatística descritiva e apresentados em tabelas na forma de frequência absoluta e percentual. Na estatística descritiva, foram elaborados tabelas e gráficos das quantidades e porcentagens das variáveis não numéricas e para as variáveis numéricas (variável idade) foram apresentadas as médias e os respectivos desvios padrão.

Para a verificação da existência de diferença estatística significativa entre as proporções de cada categoria das variáveis utilizou-se o teste Qui-quadrado, que mede se existe diferença entre as proporções das variáveis de uma tabela de dupla entrada com mais de duas categorias. Para o 
caso de variáveis com apenas duas categorias foi utilizado o teste binomial, que tem a mesma função do teste de qui-quadrado.

O teste de Kruskal-Wallis é um teste de hipóteses não paramétricos, usado para comparar três ou mais categorias. É utilizado para testar a hipótese nula de que todas as categorias têm a mesma média contra a hipótese alternativa de que pelo menos duas delas têm diferentes médias. O Teste de Wilcoxon-Mann-Whitney é um método alternativo não paramétrico do teste-t para duas categorias que é usado para testar se as medianas das categorias são iguais.

As análises estatísticas foram realizadas no programa SPSS 21.0 , sendo considerado como estatisticamente significante quando a probabilidade for menor ou igual a 0,05 ( $p$-valor $<0,05$ ).

\section{RESULTADOS}

Dos 50 participantes do estudo, 43 (86\%) eram do sexo feminino e apenas 7 (14\%) eram do sexo masculino. Observa-se uma diferença significativa nas proporções das categorias de sexo, de acordo com o teste binomial.

Nos achados deste estudo encontrou-se uma maior prevalência de litíase biliar na faixa etária de 31 a 60 anos, como observado na tabela 1.

Tabela 1. Prevalência de colelitíase segundo faixa etária

\begin{tabular}{lcc}
\hline \hline $\begin{array}{l}\text { Faixa etária } \\
\text { (Média } \pm \text { Desvio padrão: } 48 \pm 15 \text { anos) }\end{array}$ & $\mathbf{n}$ & $\%$ \\
\hline Até 30 anos & 7 & 14,0 \\
De 31 a 60 anos & 33 & 66,0 \\
Acima de 60 anos & 10 & 20,0 \\
\hline Total & $\mathbf{5 0}$ & $\mathbf{1 0 0 , 0}$ \\
\hline \hline
\end{tabular}

Teste Qui-Quadrado, p-valor: $<0,001$

Em relação ao estado nutricional observa-se uma diferença significativa $(p<0,001)$ entre as classificações do IMC (Tabela 2)

Tabela 2. Estado nutricional através do IMC em portadores de colelitiase

\begin{tabular}{lcc}
\hline \hline IMC & $\mathbf{n}$ & $\mathbf{\%}$ \\
\hline Abaixo do peso & 1 & 2,0 \\
Eutrófico & 2 & 4,0 \\
Sobrepeso & 2 & 4,0 \\
Obesidade & 45 & 90 \\
\hline Total & $\mathbf{5 0}$ & $\mathbf{1 0 0 , 0}$ \\
\hline \hline
\end{tabular}

Teste Qui-Quadrado, p-valor: $<0,001$

Quando analisado o QFA percebeu-se que $94 \%$ dos participantes consumiam pelo menos um alimento considerado como promotor de colelitíase duas vezes ao dia e $54 \%$ da amostra consumia pelo menos 3 alimentos considerados como promotores de colelitíase duas vezes ao dia, conforme apresentado na Tabela 3. 
Tabela 3. Quantidade de Alimentos Promotores de Colelitíase Ingeridos duas vezes por dia.

\begin{tabular}{ccc}
\hline \hline $\begin{array}{c}\text { Quantidade de } \\
\text { alimentos }\end{array}$ & $\mathbf{n}$ & $\mathbf{\%}$ \\
\hline 0 & 3 & $6,0 \%$ \\
1 & 9 & $18,0 \%$ \\
2 & 11 & $22,0 \%$ \\
3 & 6 & $12,0 \%$ \\
4 & 7 & $14,0 \%$ \\
5 & 6 & $12,0 \%$ \\
6 & 3 & $6,0 \%$ \\
7 & 3 & $6,0 \%$ \\
8 & 1 & $2,0 \%$ \\
13 & 1 & $2,0 \%$ \\
\hline Total & $\mathbf{5 0}$ & $\mathbf{1 0 0 , 0}$ \\
\hline \hline
\end{tabular}

\section{DISCUSSÃO}

A maior prevalência no sexo feminino encontrado neste estudo justifica-se pelo perfil hormonal da mulher, pois o hormônio estrogênio aumenta o risco de cálculos de colesterol, por aumentar a secreção de colesterol e diminuir a secreção de ácidos biliares, além de implicar na redução da motilidade da vesícula biliar ${ }^{16}$.

Os dados encontrados no presente estudo com relação ao sexo vão de acordo com os resultados de Menezes et al. ${ }^{15}$ que encontraram $80 \%$ do sexo feminino e $20 \%$ do sexo masculino de um total de 40 participantes submetidos ao seu estudo.

Ao analisarmos a faixa etária, uma possível justificativa para a maior incidência na faixa etária de 31 à 60 anos é que este intervalo de etário compreende o período da mulher fértil, no qual há uma maior produção de estrogênio endógeno além de o uso de contraceptivos ser mais recorrente, e o início da menopausa, quando muitas mulheres iniciam a terapia de reposição hormonal. Uma sugestão para os próximos estudos seria observar nas mulheres portadoras de litíase biliar, no início da menopausa, se fazem terapia de reposição hormonal e qual tipo de hormônio utilizam.

A obesidade é considerada um fator de risco para a colelitíase devido ao aumento da saturação de colesterol na bile, que amplia a propensão da formação de cálculos de colesterol ${ }^{17}$. 0 que justifica um maior número de obesos dentro da amostra.

Dentro da amostra, o consumo de alimentos promotores de colelitíase (gordurosos e ricos em açúcares) foram consumidos em grande quantidade. É sabido que o alto consumo de alimentos ricos em gorduras, açúcares e alimentos refinados são fatores importantes que podem induzir a obesidade, levando também a uma dislipidemia e a síndrome metabólica, estas condições levam a uma supersaturação de colesterol na bile e a uma diminuição da motilidade da vesícula, levando assim a formação de cálculos biliares ${ }^{9,18}$. Dessa forma, os achados do estudo vão de encontro com as informações encontradas na literatura a despeito da influência da alimentação na litogênese.

\section{CONCLUSÃO}

Os dados encontrados no presente estudo corroboram com os dados da literatura, pois em virtude dos fatos e premissas expostas, fica evidente que a maior incidência de colelitíase ocorre no sexo feminino em idade fértil (31 a 60 anos) e em indivíduos obesos. Os hábitos alimentares também podem influenciar na litogênese visto que uma alimentação rica em gorduras e açúcares simples podem favorecê-la. 


\section{REFERÊNCIAS}

1. Fauci A, Baunwald E, Kasper D, Hauser S, Longo D, Jameson L; Loscalzo J. Harrison Medicina Interna. 17 ed. Rio de Janeiro: McGraw-Hill Interamericana do Brasil, 2008. 2 v.

2. Coelho JCU, Freitas AT, Fontan RS, Campos ACL, Zeni NC, Oliva LV. Incidência de colesterolose da vesicula biliar em autopsias. Rev Col Bras Cir.1993;20:295-7.

3. Torres OJM, Barbosa ES, Pantoja PB, Diniz MC, Silva JRS, Czecko NG. Prevalência ultrasonográfica de litíase biliar em pacientes ambulatoriais. Rev. Col. Bras. Cir., São Luis MA, v. 32, n. 1, p. 47-49. 07/2004.

4. Robbins SL, Cotran RS. Patologia: Bases patológicas da doença. 8ª ed. Rio de Janeiro: Elsevier, 2010.

5. Sousa KPQ, Souza PM, Guimarães NG. Fatores antropométricos, bioquímicos e dietéticos envolvidos na litíase biliar. Com. Ciências Saúde, Brasilia - Df, v. 19, n. 3, p.261-270, jul. 2008.

6. Goldman L, Ausiello D. Cecil Medicina. 23 ed. Rio de Janeiro: Elsevier, 2009, 2v.

7. Raymond JL, Mahan K, Escott-Stump S. Krause Alimentos, Nutrição e Dietoterapia. 13ạ ed. Rio de Janeiro: Elsevier, 2013.

8. Yoo, Eun-hyung, Lee, Soo-youn. The prevalence and risk factors for gallstones disease. Clin Chem Lab Med, New York, v. 47, n. 7, p.795-807, jan. 2009.

9. Melo, MTPT. Litíase Vesicular. Fisiopatologia, diagnóstico e tratamento. 2012. 21 f. Monografia (Especialização) - Curso de Mestrado Integrado em Medicina, Departamento de Cirurgia Geral, Faculdade de Medicina da Universidade do Porto, Portugal, 2012.

10. WHO. World Health Organization. Obesity Preventing and managing the global epidemic: report of a WHO Consultation on Obesity. Geneva: WHO; 1998.

11. Lipschitz DA. Screening for nutritional status in the elderly. Prim Care. 1994;22:55-67.

12. Ribeiro AC, Savio KEO, Rodrigues MLCF; Costa THM, Schmitz BAS. Validação de um questionário de frequência de consumo alimentar para população adulta. Revista de Nutrição, Campinas, v. 19, n.5, p. 553-562, set./out. 2006, https://doi.org/10.1590/S1415-

$\underline{52732006000500003}$.

13. Sichieri R, Everhart JE. Validity of a Brazilian food frequency questionnaire against dietary recalls and estimated energy intake. Nutrition Research, v. 18, n. 10, p. 1649-1659, 1998, https://doi.org/10.1016/S0271-5317(98)00151-1.

14. Vieira S. Bioestatística Tópicos Avançados. 3 ed. Elsevier Brasil, 2011. 288 p.

15. Menezes HL, Fireman PA, Wanderley VE, Mendonça AMC, Bispo RKA, Reis MR. Estudo randomizado para avaliação da dieta hipolipidídica nos sintimas digestivos no pós-operatório imediato da colecistectomia por vídeolaparoscopia. Ver. Col. Bras. Cir., Rio de Janeiro, v. 40, n. 3, p. 203-207, Junho 2013. 
16. Wang HH, Liu M, Clegg DJ, Portincasa $P$, Wang DQ. New insights into the molecular mechanisms underlying effects of estrogen os cholesterol gallstone formation. Biochimica Et Biophysica Acta (bba) - Molecular And Cell Biology Of Lipids, [s.I.], v.1791, n.11, p. 1037-1047, nov. 2009. Elsevier BV. http://dx.doi.org/10.1016/i.bbalip.2009.06.006

17. Stender S, Nordestgaard BG, Tybjaerg-Hansen A. Elevated mass index as a casual risk fator for symptomatic gallstone disease: a mendelian randomization study. Hepatology, [s.l.], v. 58, n. 6, p.2133-2141, 11 out. 2013. Wiley-Blackwell. http://dx.doi.org/10.1002/hep.26563.

18. Tincôco ALA, Cruz MC. Hipertensão arterial e Obesidade: Luta pela Universalização do conhecimento das doenças crônicas não transmissíveis. Agência brasileira do ISBN, v. 12, 2006. 